Stud. Univ. Babeş-Bolyai Math. 66(2021), No. 2, 297-305

DOI: 10.24193/subbmath.2021.2.06

\title{
Barbashin conditions for uniform instability of evolution operators
}

\author{
Mihail Megan and Rovana Boruga (Toma)
}

Dedicated to Professor Gheorghe Coman on the occasion of his 85th anniversary.

\begin{abstract}
The aim of the present paper is to give some characterization theorems of Barbashin type for the uniform exponential instability and uniform polynomial instability behavior of evolution operators. Also, some examples which illustrate the connections between the concepts presented are given.
\end{abstract}

Mathematics Subject Classification (2010): 47B01, 34D05.

Keywords: Evolution operator, uniform instability, Barbashin conditions.

\section{Introduction}

In the last period significant progress has been made in the study of exponential stability, dichotomy and trichotomy in Banach spaces. A great number of papers that describe the asymptotic behavior of evolution operators in the exponential case was published, see for example [7], [8], [9], [11] and the references therein. In particular, the uniform exponential instability was studied in [5], [4], [12], [13], [15].

Later, the need for a new approach arose from the fact that in some situations, in particular for nonautonomous systems, the exponential stability is too stringent. In this sense a polynomial asymptotic behavior was introduced by L. Barreira and C. Valls ([2]) for the continuous case, respectively by A.J.G. Bento and C.M.Silva ([3]) for discrete-time systems.

Also, another interesting idea in this area can be found in [10] where A.L. Sasu, M. Megan and B. Sasu give some theorems of characterization for the concept of uniform exponential instability in terms of Banach function spaces. Recently, the same authors proposed in [14] an overview in the framework of Banach sequence spaces and their applications in the asymptotic theory of variational equations.

In this paper we focus on the concepts of uniform exponential instability, *uniform exponential instability, uniform polynomial instability and *-uniform polynomial instability for evolution operators in Banach spaces. 
We obtain some characterization theorems of Barbashin type ([1]) for the concepts mentioned above, assuming that the evolution operator has exponential decay, *-exponential decay, respectively polynomial decay, *-polynomial decay. Also, we establish the connections between the notions defined in the paper and the decay properties, by giving some illustrative examples in this sense.

\section{Preliminaries}

Let $X$ be a real or complex Banach space and $X^{*}$ its dual space. We denote by $\mathcal{B}(X)$ the Banach algebra of all bounded linear operators acting on $X$. We denote by $I$ the identity operator and the norms on $X, X^{*}$ and on $\mathcal{B}(X)$ will be denoted by $\|$.$\| .$ By $\Delta$ and $T$ we will denote the following sets

$$
\Delta=\left\{(t, s) \in \mathbb{R}_{+}^{2}: t \geq s\right\}, \quad T=\left\{\left(t, s, t_{0}\right) \in \mathbb{R}_{+}^{3}: t \geq s \geq t_{0}\right\} .
$$

Definition 2.1. An application $U: \Delta \rightarrow \mathcal{B}(X)$ is said to be an evolution operator on $X$ if the following relations are satisfied:

$\left(e_{1}\right) U(t, t)=I$ for all $t \geq 0$

$\left(e_{2}\right) U(t, s) U\left(s, t_{0}\right)=U\left(t, t_{0}\right)$ for all $\left(t, s, t_{0}\right) \in T$. In addition,

$\left(e_{3}\right)$ if for all $(t, s) \in \Delta$ the linear operator $U(t, s)$ is bijective then we say

that the evolution operator $U$ is reversible.

In this case, we denote by $V: \Delta \rightarrow \mathcal{B}(X)$ the inverse of the evolution operator $U$, which means that $V(t, s)=U(t, s)^{-1}$.

Remark 2.2. If $U: \Delta \rightarrow \mathcal{B}(X)$ is a reversible evolution operator, then the following properties hold:

(i) $V(t, t)=I$ for all $t \geq 0$

(ii) $V\left(t, t_{0}\right)=V\left(s, t_{0}\right) V(t, s)$ for all $\left(t, s, t_{0}\right) \in T$.

Definition 2.3. An evolution operator $U: \Delta \rightarrow \mathcal{B}(X)$ is said to be strongly measurable if for all $(s, x) \in \mathbb{R}_{+} \times X$, the mapping $t \mapsto\|U(t, s) x\|$ is measurable on $[s, \infty)$.

Definition 2.4. An evolution operator $U: \Delta \rightarrow \mathcal{B}(X)$ is said to be $*$-strongly measurable if for all $\left(s, x^{*}\right) \in \mathbb{R}_{+} \times X^{*}$, the mapping $t \mapsto\left\|U(t, s)^{*} x^{*}\right\|$ is measurable on $[0, t)$.

Definition 2.5. The evolution operator $U: \Delta \rightarrow \mathcal{B}(X)$ has uniform exponential decay (u.e.d.) if there exist two constants $M \geq 1$ and $\omega>0$ such that:

$$
\|x\| \leq M e^{\omega(t-s)}\|U(t, s) x\|, \text { for all }(t, s, x) \in \Delta \times X
$$

Definition 2.6. The evolution operator $U: \Delta \rightarrow \mathcal{B}(X)$ is said to be uniformly exponentially instable (u.e.is.) if there exist $N \geq 1$ and $\nu>0$ such that:

$$
\|x\| \leq N e^{-\nu(t-s)}\|U(t, s) x\|, \text { for all }(t, s, x) \in \Delta \times X .
$$


Definition 2.7. The evolution operator $U: \Delta \rightarrow \mathcal{B}(X)$ has uniform exponential growth (u.e.g.) if there exist two constants $M \geq 1$ and $\omega>0$ such that:

$$
\|U(t, s) x\| \leq M e^{\omega(t-s)}\|x\|, \text { for all }(t, s, x) \in \Delta \times X .
$$

Definition 2.8. The evolution operator $U: \Delta \rightarrow \mathcal{B}(X)$ is said to be uniformly exponentially stable (u.e.s.) if there exist $N \geq 1$ and $\nu>0$ such that:

$$
\|U(t, s) x\| \leq N e^{-\nu(t-s)}\|x\|, \text { for all }(t, s, x) \in \Delta \times X .
$$

Definition 2.9. The evolution operator $U: \Delta \rightarrow \mathcal{B}(X)$ has uniform polynomial decay (u.p.d.) if there exist two constants $M \geq 1$ and $\omega>0$ such that:

$$
(s+1)^{\omega}\|x\| \leq M(t+1)^{\omega}\|U(t, s) x\| \text {, for all }(t, s, x) \in \Delta \times X .
$$

Definition 2.10. The evolution operator $U: \Delta \rightarrow \mathcal{B}(X)$ is uniformly polynomially instable (u.p.is.) if there exist $N \geq 1$ and $\nu>0$ such that:

$$
(t+1)^{\nu}\|x\| \leq N(s+1)^{\nu}\|U(t, s) x\|, \text { for all }(t, s, x) \in \Delta \times X .
$$

Definition 2.11. The evolution operator $U: \Delta \rightarrow \mathcal{B}(X)$ has *-uniform exponential decay (*-u.e.d.) if there exist two constants $M \geq 1$ and $\omega>0$ such that:

$$
\left\|x^{*}\right\| \leq M e^{\omega(t-s)}\left\|U(t, s)^{*} x^{*}\right\|, \text { for all }\left(t, s, x^{*}\right) \in \Delta \times X^{*} .
$$

Definition 2.12. The evolution operator $U: \Delta \rightarrow \mathcal{B}(X)$ is said to be *-uniformly exponentially instable (*-u.e.is.) if there exist $N \geq 1$ and $\nu>0$ such that:

$$
\left\|x^{*}\right\| \leq N e^{-\nu(t-s)}\left\|U(t, s)^{*} x^{*}\right\| \text {, for all }\left(t, s, x^{*}\right) \in \Delta \times X^{*} .
$$

Definition 2.13. The evolution operator $U: \Delta \rightarrow \mathcal{B}(X)$ has *-uniform polynomial decay (*-u.p.d.) if there exist $M \geq 1$ and $\omega>0$ such that:

$$
(s+1)^{\omega}\left\|x^{*}\right\| \leq M(t+1)^{\omega}\left\|U(t, s)^{*} x^{*}\right\| \text {, for all }\left(t, s, x^{*}\right) \in \Delta \times X^{*} .
$$

Definition 2.14. The evolution operator $U: \Delta \rightarrow \mathcal{B}(X)$ is *-uniformly polynomially instable (*-u.p.is.) if there exist $N \geq 1$ and $\nu>0$ such that:

$$
(t+1)^{\nu}\left\|x^{*}\right\| \leq N(s+1)^{\nu}\left\|U(t, s)^{*} x^{*}\right\| \text {, for all }\left(t, s, x^{*}\right) \in \Delta \times X^{*} .
$$

Remark 2.15. Let $U: \Delta \rightarrow \mathcal{B}(X)$ be a reversible evolution operator. Then, $U$ has uniform exponential decay if and only if $V$ has uniform exponential growth.

Remark 2.16. Let $U: \Delta \rightarrow \mathcal{B}(X)$ be a reversible evolution operator. Then, $U$ is uniformly exponentially instable if and only if $V$ is uniformly exponentially stable.

Remark 2.17. The following diagram illustrates the connections between the instability concepts and the decay properties mentioned in the paper.

$$
\begin{array}{ccc}
\text { u.e.is. } & \Rightarrow & \text { u.p.is. } \\
\Downarrow & & \Downarrow \\
\text { u.e.d. } & \Leftarrow & \text { u.p.d. }
\end{array}
$$

In general, the converse implications are not true. Next, we will present some examples which clarify the relations given above. 
Example 2.18. (Evolution operator which has u.e.d, but it is not u.e.is.)

Let $X=\mathbb{R}$ and $U: \Delta \rightarrow \mathcal{B}(\mathbb{R}), U(t, s) x=e^{s-t} x$.

Then, $U$ has uniform exponential decay, but it is not uniformly exponentially instable. Indeed, we have that

$$
\|U(t, s)\|=e^{s-t}=e^{-(t-s)} \geq e^{-2(t-s)},
$$

which implies that $U$ u.e.d. for $\omega=2, M=1$.

If we suppose that $U$ is u.e.is., then there exist $N \geq 1, \nu>0$ such that $e^{\nu(t-s)} \leq N e^{s-t}$, for all $(t, s) \in \Delta$.

For $s=0$ and $t \rightarrow \infty$ it results that $\infty \leq N$, contradiction.

Example 2.19. (Evolution operator which is u.p.is., but it is not u.e.is.)

We consider $X=\mathbb{R}$ and the application $u:[1, \infty) \rightarrow \mathbb{R}_{+}^{*}, u(t)=t^{2}+1$. Then

$$
U: \Delta \rightarrow \mathcal{B}(\mathbb{R}), U(t, s) x=\frac{u(t)}{u(s)} x,
$$

is an evolution operator which is uniformly polynomially instable, but it is not uniformly exponentially instable.

Proof. See [13].

Example 2.20. (Evolution operator which has u.p.d., but it is not u.p.is.)

We consider $X=\mathbb{R}$ and the evolution operator

$$
\begin{gathered}
U: \Delta \rightarrow \mathcal{B}(\mathbb{R}), U(t, s) x=\frac{\varphi(s)}{\varphi(t)} x, \text { where } \\
\varphi: \mathbb{R}_{+} \rightarrow[1, \infty) \varphi(t)=t+1 .
\end{gathered}
$$

Then, $U$ has uniform polynomial decay, but it is not uniformly polynomially instable. Indeed, if we compute the norm of the operator $U$ we obtain immediately that $U$ has u.p.d. for $M=\omega=1$.

If we suppose that $U$ is u.p.is., then there exist $N \geq 1$ and $\nu>0$ such that

$$
\left(\frac{t+1}{s+1}\right)^{\nu} \leq N\left(\frac{s+1}{t+1}\right), \text { for all } t \geq s \geq 0
$$

For $s=0$ we obtain $(t+1)^{\nu+1} \leq N$, which for $t \rightarrow \infty$ yields to a contradiction.

Example 2.21. (Evolution operator that has u.e.d., but not u.p.d.)

We consider the evolution operator given in Example 2.18. We have that

$$
\|U(t, s)\|=e^{s-t} \geq e^{-2(t-s)},
$$

which implies that $U$ has uniform exponential decay for $M=1$ and $\omega=2$.

If we suppose that $U$ has u.p.d., it results that there exist $M \geq 1$ and $\omega>0$ such that

$$
\left(\frac{s+1}{t+1}\right)^{\omega} \leq M e^{s-t}, \text { for all }(t, s) \in \Delta .
$$

For $s=0$ we obtain $M \geq \frac{e^{t}}{(t+1)^{\omega}}$ which for $t \rightarrow \infty$ yields to a contradiction, so $U$ has not uniform polynomial decay. 
We define $U_{1}: \Delta \rightarrow B(X), U_{1}(t, s)=U\left(e^{t}-1, e^{s}-1\right)$ the evolution operator associated to $U$.

Proposition 2.22. The evolution operator $U: \Delta \rightarrow \mathcal{B}(X)$ has *-uniform polynomial decay if and only if the evolution operator $U_{1}: \Delta \rightarrow \mathcal{B}(X)$ has *-uniform exponential decay.

Proof. It results in a similar manner as Proposition 2.12 from [6].

Proposition 2.23. The evolution operator $U: \Delta \rightarrow \mathcal{B}(X)$ is $*$-uniformly polynomially instable if and only if $U_{1}: \Delta \rightarrow \mathcal{B}(X)$ is *-uniformly exponentially instable.

Proof. It follows using analogous arguments with those used to prove Proposition 2.13 in $[6]$.

\section{The main results}

The results of this section are some characterization theorems of Barbashin type for the uniform exponential instability, *-uniform exponential instability, respectively for the uniform polynomial instability and $*$-uniform polynomial instability for evolution operators in Banach spaces.

Theorem 3.1. Let $U$ be a *-strongly measurable evolution operator with $*$-uniform exponential decay. Then $U$ is *-uniformly exponentially instable if and only if there exist the constants $B>1$ and $b>0$ such that

$$
\int_{0}^{t} \frac{e^{-b s}}{\left\|U(t, s)^{*} x^{*}\right\|} d s \leq \frac{B e^{-b t}}{\left\|x^{*}\right\|},
$$

for all $\left(t, x^{*}\right) \in \mathbb{R}_{+} \times\left(X^{*} \backslash\{0\}\right)$.

Proof. Necessity. Let $b \in(0, \nu)$. We suppose that $U$ is $*$-uniformly exponentially instable. Then, there exist $N \geq 1, \nu>0$ such that

$$
e^{\nu(t-s)}\left\|x^{*}\right\| \leq N\left\|U(t, s)^{*} x^{*}\right\| \text {, for all }\left(t, s, x^{*}\right) \in \Delta \times X^{*},
$$

which is equivalent to

$$
\begin{gathered}
\frac{1}{\left\|U(t, s)^{*} x^{*}\right\|} \leq \frac{N e^{-\nu(t-s)}}{\left\|x^{*}\right\|}, \text { for all }\left(t, s, x^{*}\right) \in \Delta \times X^{*}, \text { that implies } \\
\int_{0}^{t} \frac{e^{-b s}}{\left\|U(t, s)^{*} x^{*}\right\|} d s \leq \frac{N}{\left\|x^{*}\right\|} \int_{0}^{t} e^{-b s} \cdot e^{-\nu(t-s)} d s=\frac{N e^{-\nu t}}{\left\|x^{*}\right\|} \int_{0}^{t} e^{(\nu-b) s} d s \leq \frac{B e^{-b t}}{\left\|x^{*}\right\|},
\end{gathered}
$$


where $B=1+\frac{N}{\nu-b}$.

Sufficiency. For $t \geq s+1$ we have

$$
\begin{aligned}
\frac{e^{-b s}}{\left\|U(t, s)^{*} x^{*}\right\|} & =\int_{s}^{s+1} \frac{e^{-b s}}{\left\|U(t, s)^{*} x^{*}\right\|} d \tau=\int_{s}^{s+1} \frac{e^{-b s}}{\left\|U(\tau, s)^{*} U(t, \tau)^{*} x^{*}\right\|} d \tau \\
& \leq \frac{1}{M} \int_{s}^{s+1} \frac{e^{-b s} \cdot e^{\omega(\tau-s)}}{\left\|U(t, \tau)^{*} x^{*}\right\|} d \tau=\frac{e^{\omega}}{M} \int_{s}^{s+1} e^{b(\tau-s)} \cdot \frac{e^{-b \tau}}{\left\|U(t, \tau)^{*} x^{*}\right\|} d \tau \\
& \leq \frac{e^{\omega+b}}{M} \int_{0}^{t} \frac{e^{-b \tau}}{\left\|U(t, \tau)^{*} x^{*}\right\|} d \tau \leq \frac{B e^{\omega+b}}{M} \cdot \frac{e^{-b t}}{\left\|x^{*}\right\|} \leq N_{1} \frac{e^{-b t}}{\left\|x^{*}\right\|},
\end{aligned}
$$

where $N_{1}=1+\frac{B e^{\omega+b}}{M}$. So, we obtained that

$$
e^{b(t-s)}\left\|x^{*}\right\| \leq N_{1}\left\|U(t, s)^{*} x^{*}\right\|, \text { for all } t \geq s+1, s \geq 0 .
$$

For $t \in[s, s+1]$ we apply the $*$-decay property and we obtain that

$$
\begin{gathered}
\left\|U(t, s)^{*} x^{*}\right\| \geq M e^{-\omega(t-s)}\left\|x^{*}\right\|, \text { which implies } \\
e^{b(t-s)}\left\|x^{*}\right\| \leq \frac{e^{(\omega+b)(t-s)}}{M}\left\|U(t, s)^{*} x^{*}\right\| \leq \frac{e^{\omega+b}}{M}\left\|U(t, s)^{*} x^{*}\right\| \leq N_{2}\left\|U(t, s)^{*} x^{*}\right\|,
\end{gathered}
$$

where $N_{2}=1+\frac{e^{\omega+b}}{M}$. So, we have that

$$
e^{b(t-s)}\left\|x^{*}\right\| \leq N_{2}\left\|U(t, s)^{*} x^{*}\right\|, \text { for all } t \in[s, s+1], s \geq 0
$$

From (3.1) and (3.2) we obtain that

$$
e^{b(t-s)}\left\|x^{*}\right\| \leq N\left\|U(t, s)^{*} x^{*}\right\|, \text { for all }(t, s, x) \in \Delta \times X,
$$

where $N=\max \left\{N_{1}, N_{2}\right\}$, so the theorem is proved.

Corollary 3.2. Let $U$ be a *-strongly measurable evolution operator with *-uniform polynomial decay. Then $U$ is *-uniformly polynomially instable if and only if there exist the constants $B>1$ and $b>0$ such that

$$
\int_{0}^{t} \frac{(s+1)^{-b-1}}{\left\|U(t, s)^{*} x^{*}\right\|} d s \leq \frac{B(t+1)^{-b}}{\left\|x^{*}\right\|},
$$

for all $\left(t, x^{*}\right) \in \mathbb{R}_{+} \times\left(X^{*} \backslash\{0\}\right)$.

Proof. If $U$ is $*$-uniformly polynomially instable with $*$-uniform polynomial decay, then, from Proposition 2.22 and Proposition 2.23, this is equivalent to $U_{1}$ is $*-$ uniformly exponentially instable with $*$-uniform exponential decay, which is equivalent from Theorem 3.1 that there exist $B>1$ and $b>0$ such that

$$
\int_{0}^{t} \frac{e^{-b s}}{\left\|U\left(e^{t}-1, e^{s}-1\right)^{*} x^{*}\right\|} d s \leq \frac{B e^{-b t}}{\left\|x^{*}\right\|},
$$


for all $\left(t, x^{*}\right) \in \mathbb{R}_{+} \times\left(X^{*} \backslash\{0\}\right)$. Using the change of variables $e^{s}-1=u$ relation (3.3) is equivalent to

$$
\int_{0}^{e^{t}-1} \frac{e^{-b \ln (1+u)}}{\left\|U\left(e^{t}-1, u\right)^{*} x^{*}\right\|} \frac{d u}{1+u} \leq \frac{B e^{-b t}}{\left\|x^{*}\right\|} .
$$

Denoting by $v=e^{t}-1$ relation (3.4) becomes

$$
\int_{0}^{v} \frac{(1+u)^{-b-1}}{\left\|U(v, u)^{*} x^{*}\right\|} d u \leq \frac{B(v+1)^{-b}}{\left\|x^{*}\right\|},
$$

so we conclude that the proof is complete.

Theorem 3.3. Let $U: \Delta \rightarrow \mathcal{B}(X)$ be a strongly measurable and reversible evolution operator with uniform exponential decay. Then $U$ is uniformly exponentially instable if and only if there exist $B>1$ and $b \in(0,1)$ such that

$$
\int_{0}^{t} \frac{\|V(t, s) x\|}{e^{b s}} d s \leq \frac{B\|x\|}{e^{b t}}, \text { for all }(t, x) \in \mathbb{R}_{+} \times X .
$$

Proof. If $U: \Delta \rightarrow \mathcal{B}(X)$ is a reversible evolution operator with uniform exponential decay, then from Remark 2.15 we have that $V: \Delta \rightarrow \mathcal{B}(X)$ has uniform exponential growth.

Necessity. Let $b \in(0, \nu)$. We suppose that $U$ is uniformly exponentially instable which implies from Remark 2.16 that $V$ is uniformly exponentially stable. Then, we have

$$
\begin{aligned}
\int_{0}^{t} \frac{\|V(t, s) x\|}{e^{b s}} d s & \leq N \int_{0}^{t} \frac{e^{-\nu(t-s)}}{e^{b s}}\|x\| d s=N e^{-\nu t}\|x\| \int_{0}^{t} e^{(\nu-b) s} d s \\
& =\frac{N}{\nu-b}\|x\|\left(e^{-b t}-e^{-\nu t}\right) \leq \frac{B}{e^{b t}}\|x\|,
\end{aligned}
$$

where $B=1+\frac{N}{\nu-b}$.

Sufficiency. If $t \geq s+1$ we obtain

$$
\begin{aligned}
e^{b t}\|V(t, s) x\| & =\int_{s}^{s+1} e^{b t}\|V(\tau, s) V(t, \tau) x\| d \tau \leq M \int_{s}^{s+1} e^{b t} e^{\omega(\tau-s)}\|V(t, \tau) x\| d \tau \\
& =M \int_{s}^{s+1} e^{(\tau-s)(b+\omega)} e^{b(t+s)} \frac{\|V(t, \tau) x\|}{e^{b \tau}} d \tau \\
& \leq M e^{b+\omega} e^{b(t+s)} \int_{0}^{t} \frac{\|V(t, \tau) x\|}{e^{b \tau}} d \tau \leq N e^{b s}\|x\|
\end{aligned}
$$


where $N=B M e^{b+\omega}$.

So, we obtained

$$
\|V(t, s) x\| \leq N e^{-b(t-s)\|x\|}, \text { for all } t \geq s+1, s \geq 0 .
$$

If $t \in[s, s+1)$ we apply the growth property and we have

$$
e^{b t}\|V(t, s) x\| \leq M e^{b t} e^{\omega(t-s)}\|x\|=M e^{(b+\omega)(t-s)} e^{b s}\|x\| \leq N e^{b s}\|x\|,
$$

which implies

$$
\|V(t, s) x\| \leq N e^{-b(t-s)\|x\|}, \text { forall } t \in[s, s+1), s \geq 0 .
$$

Finally, from (3.6) and (3.7) we obtain that $V$ is uniformly exponentially stable which means from Remark 2.16 that $U$ is uniformly exponentially instable.

Corollary 3.4. Let $U: \Delta \rightarrow \mathcal{B}(X)$ be a strongly measurable and reversible evolution operator with uniform polynomial decay. Then $U$ is uniformly polynomially instable if and only if there exist $B>1$ and $b \in(0,1)$ such that

$$
\int_{0}^{t} \frac{\|V(t, s) x\|}{(s+1)^{b+1}} d s \leq \frac{B\|x\|}{(t+1)^{b}}, \text { for all }(t, x) \in \mathbb{R}_{+} \times X .
$$

Proof. It results immediately using the same idea as in the proof of Corollary 3.2.

\section{References}

[1] Barbashin, E.A., Introduction in the Theory of Stability, Nauka, Moscow, 1967.

[2] Barreira, L., Valls, C., Polynomial growth rates, Nonlinear Anal., 71(2009), 5208-5219.

[3] Bento, A., Silva, C., Stable manifolds for nonuniform polynomial dichotomies, J. Funct. Anal., 257(2009), 122-148.

[4] Biriş, L., On uniform exponential instability property of evolution operators in Banach spaces, An. Univ. Vest Timiş. Ser. Mat.-Inform., 47(2009), no. 1, 3-9.

[5] Boruga, R., Megan, M., On Some Concepts Regarding the Polynomial Behaviors for Evolution Operators in Banach Spaces, International Symposium Research and Education in an Innovation Era Conference, Mathematics and Computer Science, 2018, 18-24.

[6] Boruga, R., Megan, M., Datko criteria for uniform instability in Banach spaces, Stud. Univ. Babeş-Bolyai Math., 66(2021), no. 1, 115-122.

[7] Buşe, C., Megan, M., Preda, P., Prajea, M-S., The strong variant of a Barbashin theorem on stability of solutions for non-autonomous differential equations in Banach spaces, Integral Equations Operator Theory, 59(2007), 491-500.

[8] Datko, R., Uniform asymptotic stability of evolutionary processes in a Banach space, SIAM J. Math. Anal., 3(1972), 428-445.

[9] Megan, M., Sasu, A.L., Sasu, B., The Asymptotic Behavior of Evolution Families, Mirton Publishing House, 2003.

[10] Megan, M., Sasu, A.L., Sasu, B., Banach function spaces and exponential instability of evolution families, Arch. Math. (Brno), 39(2003), 277-286.

[11] Mihit, C.L., Megan, M., Integral characterizations for the $(h ; k)$-splitting of skewevolution semiflows, Stud. Univ. Babeş-Bolyai Math., 62(2017), no. 3, 353-365. 
[12] Popa, I.L., Nonuniform exponential instability for evolution operators in Banach spaces, Proc. of the $12^{\text {th }}$ Symposium of Math. and its Appl. "Politehnica" Univ. of Timişoara, November 5-7, 2009.

[13] Rămneanţu, M.L., Megan, M., Ceauşu, T., Polynomial instability of evolution operators in Banach spaces, Carpathian J. Math., 29(2013), no. 1, 77-83.

[14] Sasu, A.L., Megan, M., Sasu, B., On Rolewicz-Zabczyk techniques in the stability theory of dynamical systems, Fixed Point Theory, 13(2012), 205-236.

[15] Stoica, C., Megan, M., Uniform exponential instability of evolution operators in Banach spaces, An. Univ. Vest Timiş. Ser. Mat.-Inform., 44(2006), no. 2, 143-148.

Mihail Megan

Academy of Romanian Scientists,

Independenţei 54,

050094 Bucharest, Romania

and

West University of Timişoara,

Faculty of Mathematics and Computer Science,

Departament of Mathematics,

V. Pârvan Blv. 4,

300223 Timişoara, Romania

e-mail: mihail.megan@e-uvt.ro

Rovana Boruga (Toma)

West University of Timişoara,

Faculty of Mathematics and Computer Science,

Departament of Mathematics,

V. Pârvan Blv. 4,

300223 Timişoara, Romania

e-mail: rovanaboruga@gmail.com 